\title{
Coherent THz Phonons Driven by Light Pulses and the Sb Problem: What is the Mechanism?
}

\author{
G. A. Garrett, ${ }^{1,2}$ T. F. Albrecht, ${ }^{1,2}$ J.F. Whitaker, ${ }^{1}$ and R. Merlin ${ }^{1,2,3}$ \\ ${ }^{1}$ Center for Ultrafast Optical Science, The University of Michigan, Ann Arbor, Michigan 48109-2099 \\ ${ }^{2}$ The Harrison M. Randall Laboratory of Physics, The University of Michigan, Ann Arbor, Michigan 48109-1120 \\ ${ }^{3}$ Department of Physics, Hong Kong University of Science and Technology, Clear Water Bay, Kowloon, Hong Kong \\ (Received 23 July 1996)
}

\begin{abstract}
Femtosecond laser pulses generate in $\mathrm{Sb}$ coherent $E_{g}$ phonons at $\approx 3.4 \mathrm{THz}$, in addition to oscillations of $A_{1 g}$ symmetry accounted for by the phenomenological displacive-excitation model. Experiments agree with theoretical calculations showing that the coherent driving force in absorbing materials like $\mathrm{Sb}$ is determined by Raman processes, as in transparent media. The Raman formalism provides a unifying approach for describing light-induced motion of atoms of both impulsive and displacive character. [S0031-9007(96)01455-X]
\end{abstract}

PACS numbers: 78.47.+p, 63.20.Kr, 78.30.-j

Following recent advances in femtosecond laser technology, several groups demonstrated that the propagation of light pulses in solids is accompanied by intense lattice vibrations showing a high degree of spatial and temporal coherence [1-9]. The availability of coherent phonons at $\mathrm{THz}$ frequencies has led to a variety of suggestions for applications and experiments involving, in particular, time-domain spectroscopy of phonons using pumpprobe methods [1-9], conversion of mechanical into coherent electromagnetic energy [7], and intriguing proposals relying on photon control of the ionic motion $[10,11]$. While coherent vibrations have been produced in insulators, semiconductors, and metals using, basically, the same technique [1-9], the experiments reveal fundamental, but poorly understood differences between transparent and opaque materials [1,4]. This Letter presents a unifying mechanism for phonon generation that explains the differences.

Phenomenologically, the lattice motion is described by

$$
\frac{d^{2} \mathcal{Q}}{d t^{2}}+\Omega^{2} \mathcal{Q}=F(\mathbf{r}, t)
$$

where $\mathcal{Q}$ is a classical phonon field of frequency $\Omega$, and $F$ is the driving force [1]. In transparent media, it has been known for a long time that vibrational coherences rely on coherent (stimulated) Raman scattering (CRS) for which $F=\sum_{u v}\left(\chi_{u v}^{R} \mathcal{E}_{u} \mathcal{E}_{v}\right) / 2$. Here, $\mathcal{F}_{u}$ denotes a component of the optical pump field, $\chi_{u v}^{R} \approx \partial \chi_{u v}^{(1)} / \partial \mathcal{Q}$ is the nonlinear Raman, and $\chi_{u v}^{(1)}$ is the linear susceptibility [12]. When the pulse width $\tau_{0}$ is small compared with $\Omega^{-1}$, $F$ acts as an impulsive force giving $\mathcal{Q} \propto \sin (\Omega t)$. For absorbing substances, a unique process has so far not been identified [1]. The experimental evidence indicates that Raman selection rules are strictly obeyed (see, e.g., $[3,5,7,13])$. However, the oscillation phase varies from material to material, and relative intensities gained from time-domain measurements and spontaneous Raman scattering (RS) do not appear to correlate [4]. One of the leading proposals for explaining opaque systems is the mechanism known as displacive excitation of coherent phonons
(DECP). The DECP model, accounting, in particular, for results on semimetals [4], provides a simple explanation for driving fully symmetric modes, seemingly unrelated to Raman scattering $[4,14]$. In the DECP picture, the equilibrium positions of the ions experience a sudden shift due to coupling with photoexcited carriers created by the optical pulse. Hence, $F$ is steplike and, thus, $\mathcal{Q} \propto[1-$ $\cos (\Omega t)]$. As discussed below, our approach renders the DECP model $[4,14]$ as a particular case of resonant CRS in that the driving force generally contains both impulsive and displacive components. To test these ideas, we have performed pump-probe and conventional RS experiments reexamining the case of antimony [4]. We chose Sb as representative of a class of materials which have been reported to follow DECP, but not Raman predictions [4]. Unlike previous studies [4], we find that pulses drive not only one (fully-symmetric), but the two Raman-allowed modes, and that there is a strong correlation between time-domain and RS intensities. We also observe that the phase of the oscillation is intermediate between that of purely displacive and impulsive dynamics.

Measurements were performed on single crystals of antimony at room temperature using cleaved surfaces perpendicular to the trigonal axis. $\mathrm{Sb}$ is a group $\mathrm{Vb}$ semimetal crystallizing in the $A 7$ structure with the point group $\overline{3} m$ and two atoms per unit cell. The three phonon branches give the Raman-active $A_{1 g}$ (totally symmetric) and $E_{g}$ (doubly degenerate) modes at the center of the Brillouin zone. The corresponding Raman tensors are given in Table I. Time-domain data were obtained using a standard pump-probe setup in the reflection geometry. As a source, we used a mode-locked Ti-sapphire laser providing $80 \mathrm{fs}$ pulses centered at $815.0 \mathrm{~nm}$ at a repetition rate of $85 \mathrm{MHz}$ and $15 \mathrm{~mW}$ of average power focused to a 70- $\mu$ m-diameter spot. RS measurements were recorded in the backscattering configuration using $30 \mathrm{~mW}$ of a cw Ti:sapphire laser also tuned to $815.0 \mathrm{~nm}$, or an Ar laser operating at $514.5 \mathrm{~nm}$.

A summary of our time-domain results is shown in Fig. 1. In these traces, the relative reflected intensity of 
TABLE I. Raman tensors for $\overline{3} m$. The trigonal axis is along [001].

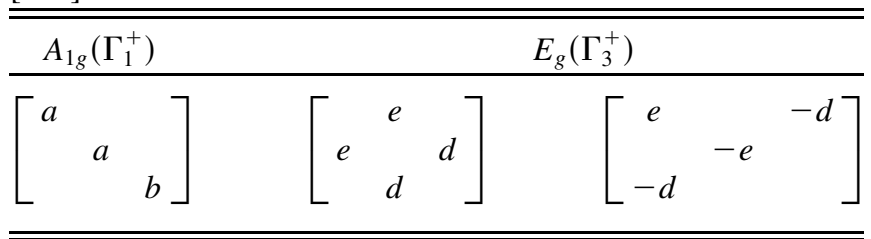

the probe beam, $\Delta R / R$, is plotted as a function of the time delay between the pump and probe pulses. Standard CRS selection rules, easily derived from Table I, dictate that the $A_{1 g}$ spectrum should not depend on $\theta$, whereas the intensity of the $E_{g}$ mode should behave as $\cos (2 \theta)$; $\theta$ is the angle between the polarizations of the two beams. Our measurements are in excellent agreement with these predictions. As illustrated in Fig. 1(a), the $\theta$ dependence of the modes allowed us to single out the $E_{g}$ contribution which could not be resolved in previous work using $\approx 2 \mathrm{eV}$ photons [4]. In Fig. 1(b), we show fits to $\exp (-\Gamma t) \sin (\Omega t+\phi)$ indicating that the dynamics of the modes are neither displacive $(\phi= \pm \pi / 2)$ nor impulsive $(\phi=0)$. This should be contrasted with the results for the $A_{1 g}$ mode at $\approx 2 \mathrm{eV}$ showing purely displacive behavior [4]. A comparison between time-
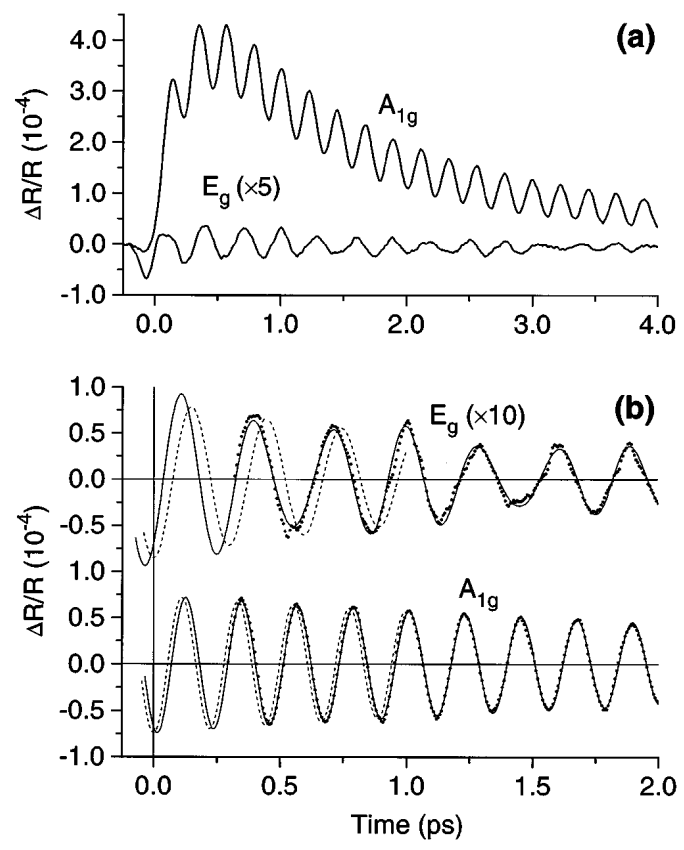

FIG. 1. Reflection geometry data. In (a), the angle between the polarizations of the two beams is $\theta=\pi / 4$ for the $A_{1 g}$ trace. $E_{g}$ results were obtained from the difference between measurements at $\theta=\pi / 2$ and at $\theta=0$. Fits to $\exp (-\Gamma t) \sin (\Omega t+\phi)$ are shown in (b), after removal of the background. Parameters are $\Omega=4.5 \mathrm{THz}, \Gamma^{-1}=3.7 \mathrm{ps}$, and $\phi=-113^{\circ} \pm 11^{\circ}$ for the $A_{1 g}$ phonon and $\Omega=3.4 \mathrm{THz}$, $\Gamma^{-1}=1.8 \mathrm{ps}$, and $\phi=-47^{\circ} \pm 8^{\circ}$ for the $E_{g}$ mode. Dashed curves are $\exp (-\Gamma t) \cos (\Omega t)$ corresponding to the displacive limit. domain and RS spectra is shown in Fig. 2. Notice that the ratio between the Fourier transform (FT) amplitudes of the two modes is very close to the RS intensity ratio, as expected for transparent substances [1]. As discussed later, this correlation supports our claim that Raman scattering is the underlying mechanism for the driving force in absorbing media. Within this context, it is important to mention that visible-range $\mathrm{RS}$ in $\mathrm{Sb}$ is dominated by the $E_{2}^{\prime}$ resonance centered at $2.15 \mathrm{eV}$ and that the ratio between $A_{1 g}$ and $E_{g}$ intensities, i.e., $|a / e|^{2}$, is nearly constant across $1.7-2.7 \mathrm{eV}$ (see Table I and the inset of Fig. 2). This indicates that two-band terms are the leading RS process [15]. From the RS data at $815.0 \mathrm{~nm}(1.52 \mathrm{eV})$, we have $|a / e|=2.2 \pm 0.5$, while, at $514.5 \mathrm{~nm}(2.41 \mathrm{eV}),|a / e|=2.4 \pm 0.6$, in reasonable agreement with [15]. Since these ratios are the same within experimental errors, we conclude that two-band processes dominate at $815.0 \mathrm{~nm}$ as well.

The simplest Hamiltonian of interest to our problem is

$$
\begin{aligned}
H= & \sum_{b k} \varepsilon_{b k} c_{b k}^{\dagger} c_{b k}+\frac{1}{2} \sum_{q}\left|P_{q}^{2}\right|+\Omega_{q}^{2}\left|Q_{q}^{2}\right| \\
& +V^{-1 / 2} \sum_{k k^{\prime}} \sum_{b b^{\prime}} \Xi_{k k^{\prime}}^{b b^{\prime}} Q_{k-k^{\prime}} c_{b k}^{\dagger} c_{b^{\prime} k^{\prime}}+V(t) .
\end{aligned}
$$

$c_{b k}^{\dagger}$ and $c_{b k}$ are electron creation and annihilation operators for the state of energy $\varepsilon_{b k}$, wave vector $\mathbf{k}$, and band

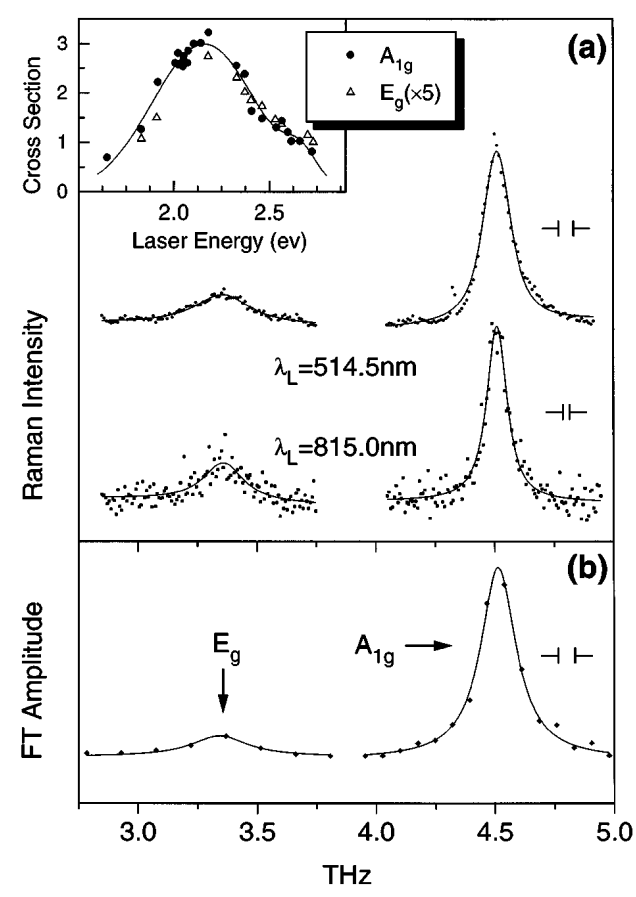

FIG. 2. Comparison between RS and time-domain results (arbitrary units). Solid lines are Lorentzian fits. (a) RS spectra for two excitation wavelengths. The polarizations of the incident and scattered light are parallel to each other. (b) FT amplitude corresponding to the traces in Fig. 1(a) obtained with pulses centered at $815 \mathrm{~nm}$. The inset of (a) shows the dependence of the RS cross section on the laser energy (from [15]). 
index $b . Q_{q}$ is the amplitude of the phonon of wave vector $q$ and frequency $\Omega_{q}$, and $P_{q}$ is the associated canonical momentum. $\Xi_{k k^{\prime}}^{b b^{\prime}}$ are matrix elements of the electron-phonon interaction and $\mathcal{V}$ is the volume. The time-dependent term describes the coupling of the system to the radiation field of the pump which we treat as classical. From the equation of motion for $Q_{q}$, we obtain the identity

$$
\frac{d^{2}\left\langle Q_{q}\right\rangle}{d t^{2}}+\Omega_{q}^{2}\left\langle Q_{q}\right\rangle=-\mathcal{V}^{-1 / 2}\left\langle\sum_{k b b^{\prime}} \Xi_{k, k-q}^{b b^{\prime}} c_{b k}^{\dagger} c_{b^{\prime} k-q}\right\rangle,
$$

leading to (1) after the replacement $Q=$ $\mathcal{V}^{-1 / 2} \sum_{q}\left\langle Q_{q}\right\rangle \exp (i \mathbf{q} \cdot \mathbf{r})$. The two-band DECP model of [14] assumes that the electronic density matrix is diagonal, i.e., that the photoinduced charge density $\rho(\mathbf{r})$ is a constant and, thus, that (3) vanishes for all wave vectors but $\mathbf{q}=0$. However, (3) can be obtained directly using perturbation theory. We show below that the electronic expectation value contains diagonal as well as off-diagonal contributions and that it is the off-diagonal terms, rather than the diagonal ones, which are responsible for the coherent oscillations. A straightforward second-order calculation gives

$$
\begin{aligned}
\left\langle\sum_{k b b^{\prime}} \Xi_{k, k-q}^{b b^{\prime}} c_{b k}^{\dagger} c_{b^{\prime} k-q}\right\rangle=\frac{\mathcal{V}}{2 \pi^{2} c^{2}} \sum_{u v} & \left(\int \mathcal{A}_{u}^{*}\left(\omega_{1}, \mathbf{k}_{1}\right) e^{i \omega_{1} t}\right. \\
& \left.\times \mathcal{R}_{u v}\left(\omega_{1}, \omega_{2}, \mathbf{q}\right) \mathcal{A}_{v}\left(\omega_{2}, \mathbf{k}_{2}\right) e^{-i \omega_{2} t} d\left(\omega_{1}^{2}\right) d\left(\omega_{2}^{2}\right) d^{3} k_{1} d^{3} k_{2}\right),
\end{aligned}
$$

where

$$
\mathcal{R}_{u v}=(e / m \hbar)^{2}\left(\mathcal{V} \omega_{1} \omega_{2}\right)^{-1}\left[r_{u v}\left(\omega_{1}, \omega_{2}, \mathbf{q}\right)+r_{v u}\left(-\omega_{2},-\omega_{1}, \mathbf{q}\right)\right]
$$

with $\left(\gamma \rightarrow 0^{+}\right)$

$$
\begin{aligned}
r_{u v}\left(\omega_{1}, \omega_{2}, \mathbf{q}\right)= & \sum_{m, n}\left(\frac{\Pi_{0 m}^{u}\left(\mathbf{k}_{1}\right) \Xi_{m n}(\mathbf{q}) \Pi_{n 0}^{v}\left(\mathbf{k}_{2}\right)}{\left[\omega_{m}+i \gamma / 2-\omega_{1}\right]\left[\omega_{n}-i \gamma / 2-\omega_{2}\right]}\right. \\
& \left.+\frac{\Xi_{0 m}(\mathbf{q}) \Pi_{m n}^{u}\left(\mathbf{k}_{1}\right) \Pi_{n 0}^{v}\left(\mathbf{k}_{2}\right)}{\left[\omega_{m}-i \gamma+\omega_{1}-\omega_{2}\right]\left[\omega_{n}-i \gamma / 2-\omega_{2}\right]}+\frac{\Xi_{0 m}(\mathbf{q}) \Pi_{m n}^{u}\left(\mathbf{k}_{2}\right) \Pi_{n 0}^{v}\left(\mathbf{k}_{1}\right)}{\left[\omega_{m}+i \gamma-\omega_{1}+\omega_{2}\right]\left[\omega_{n}+i \gamma / 2-\omega_{1}\right]}\right) .
\end{aligned}
$$

$\mathcal{A}_{u}$ are the components of the vector potential and $\Pi^{u}(\mathbf{k})=\sum_{j}\left(\hat{\mathbf{e}}_{u} \cdot \mathbf{p}_{j}\right) \exp \left(-i \mathbf{k} \cdot \mathbf{r}_{j}\right) ; \mathbf{r}_{j}$ and $\mathbf{p}_{j}$ are, respectively, the electron position and momentum, and $\hat{\mathbf{e}}_{u}$ are unit vectors perpendicular to $\mathbf{k}$. If we ignore the weak $\mathbf{q}$ dependence of $\mathcal{R}_{u v}$ and set $\Omega(\mathbf{q}) \approx \Omega$, the driving force in (1) can be written as

$$
\begin{aligned}
F(\mathbf{r}, t) \approx & -\left(1 / 8 \pi^{2}\right) \int \mathcal{E}_{0}\left(\mathbf{r}, t_{1}\right) e^{-i \omega_{1}\left(t_{1}-t\right)} \mathcal{E}_{0}\left(\mathbf{r}, t_{2}\right) e^{i \omega_{2}\left(t_{2}-t\right)} \\
& \times \sum_{u v}\left(\cos \left(\alpha_{u}\right) \mathcal{R}_{u v}\left(\omega_{1}, \omega_{2}\right) \cos \left(\alpha_{v}\right)\right) d t_{1} d t_{2} d \omega_{1} d \omega_{2},
\end{aligned}
$$

where $\cos \left(\alpha_{u}\right)=\mathcal{E}_{u} / \mathcal{E}_{0}$ and $\mathcal{E}_{0}$ is the amplitude of the field. Since $\mathcal{E}_{0}$ decays with a penetration depth given by $c /\left(\kappa \omega_{0}\right)$, it is clear that $F$ couples to a continuum of modes in the range $\delta q \sim 2 \kappa \omega_{0} / c$ about the phase matching $\mathbf{q}= \pm \Omega n \hat{\mathbf{s}} / c(n+i \kappa$ is the refractive index and $\hat{\mathbf{s}}$ is a unit vector in the direction of propagation of the light). This contradicts the DECP prediction [14], based on the assumption that the density matrix is diagonal, that photoexcitation drives only the $q=0$ mode [16].

Let $\omega_{0}$ be the central frequency of the optical pulse. The behavior of $\mathcal{Q}$ is determined primarily by the frequency components of $F$ in the vicinity of $\Omega$ and, therefore, by the properties of $\mathcal{R}_{u v}$ at $\omega_{1}-\omega_{2} \approx \pm \Omega$ and $\omega_{1} \approx \omega_{2} \approx \pm \omega_{0}$ (as opposed to $\omega_{1} \approx-\omega_{2} \pm \omega_{0}$ giving frequencies $\sim 2 \omega_{0}$ that are too high to effectively drive the oscillator). Since $\chi_{u v}^{R} \propto \mathcal{R}_{u v}(\omega, \omega-\Omega, \mathbf{q})$, this establishes the link between $F$ and the Raman susceptibility. For transparent media, we can safely replace in (6) $\mathcal{R}_{u v}$ by its (real) value at $\omega_{1}=\omega_{2}=\omega_{0}$ with the result that, for pulses of width $\tau_{0} \ll \Omega^{-1}, F \propto$ $\left|\mathcal{E}_{0}(\mathbf{r}, t)\right|^{2}$ is impulsive [1]. In absorbing substances, however, dispersive effects cannot be ignored. Consider first the term of (5) containing two resonant denominators and, in particular, two-band contributions for which the relevant matrix elements are $\Xi_{k k^{\prime}}^{b b^{\prime}}$ with $b \equiv b^{\prime}$ [17]. As mentioned earlier, these processes dominate at the $\mathrm{Sb}$ $E_{2}^{\prime}$ resonance [15]. Integrating (6) over $\omega_{1}$ and $\omega_{2}$, and expressing the field as $f(\mathbf{r}, t) \exp \left[-i \omega_{0} t+(i n-\right.$ $\left.\boldsymbol{\kappa}) k_{0} \hat{\mathbf{s}} \cdot \mathbf{r}\right]+$ c.c., where $f$ is the envelope, we find that states for which $\left|\omega_{n}-\omega_{0}\right| \gg \tau_{0}^{-1}$ provide an impulsive contribution of the form

$$
\begin{aligned}
F_{\mathrm{I}} \approx & -\frac{|f(\mathbf{r}, t)|^{2} e^{-2 \kappa k_{0} \mathbf{s} \cdot \mathbf{r}}}{V(m \hbar / e)^{2}} \\
& \times \sum_{u v, n}^{\prime}\left(1+\omega_{0}^{2} / \omega_{n}^{2}\right) \frac{\Xi_{n n}\left(\Pi_{0 n}^{u} \cos \left(\alpha_{u}\right) \Pi_{n 0}^{v} \cos \left(\alpha_{v}\right)\right)}{\left(\omega_{0}^{2}-\omega_{n}^{2}\right)^{2}},
\end{aligned}
$$


whereas frequencies satisfying $\left|\omega_{n}-\omega_{0}\right| \ll \tau_{0}^{-1}$ exhibit displacive character. In particular for $t \gg \tau_{0}, F$ approaches the exact limiting value

$$
\begin{aligned}
F_{\mathrm{D}}= & -\frac{\mathcal{V}^{-1}}{2(m \hbar / e)^{2}} \sum_{u v, n}\left|\mathcal{E}_{0}\left(\mathbf{r}, \omega_{n}\right)\right|^{2} \\
& \times \Xi_{n n}\left(\Pi_{0 n}^{u} \cos \left(\alpha_{u}\right) \Pi_{n 0}^{v} \cos \left(\alpha_{v}\right)\right) / \omega_{n}^{2},
\end{aligned}
$$

where $\mathcal{E}_{0}\left(\mathbf{r}, \omega_{n}\right)$ is the Fourier transform of $\mathcal{E}_{0}(\mathbf{r}, t)$. These expressions simply reflect the fact that phonons couple to virtual charge-density fluctuations for $\mid \omega_{n}$ $\omega_{0} \mid \gg \tau_{0}^{-1}$, but to real ones in the opposite limit [we notice from (3) that $F \propto \rho(\mathbf{r})$ if the coefficients $\Xi_{k k^{\prime}}^{b b}$ are approximated by constants, one for each band]. In the former case, the optical pulse gives an impulsive force because the fluctuations lifetime is $\sim\left|\omega_{n}-\omega_{0}\right|^{-1}$. On the other hand, and as in the DECP model $[4,14]$, real transitions lead to shifts in the equilibrium positions of the ions (the lifetime of the fluctuation is that of the Raman coherence) [18]. In the common situation where the width of the Raman resonance $\delta_{R}$ is large compared with $\tau_{0}^{-1}$, it can be shown after summation over $\omega_{n}$ that the driving force is purely displacive for $\left|\omega_{R}-\omega_{0}\right| \ll \delta_{R}$ gradually becoming impulsivelike at $\left|\omega_{R}-\omega_{0}\right| \gg \delta_{R}$ where $\omega_{R}$ is the frequency of the resonance maximum (in $\mathrm{Sb}, \delta_{R} \approx 0.3 \mathrm{eV}$ and $\omega_{R} \approx 2.15 \mathrm{eV}$; see inset of Fig. 2). It is important to stress the fact that (8) applies to Raman-active phonons of arbitrary symmetry, in contrast to the DECP model which concerns itself exclusively with fully symmetric modes [4]. We also note that, in most circumstances, three-band processes [17] and the remaining terms in (5) having at most one resonant denominator provide additional contributions to (7) but not to (8), i.e., only resonant two-band terms contribute to $F_{\mathrm{D}}$. This explains why displacive behavior is seen in certain materials like $\mathrm{Sb}$, but not in others.

Comparisons between RS and time-domain measurements of the relative intensity, and between our values for the coherent oscillator parameters and those reported in [4] strongly support the Raman interpretation. In [4], $\left|\left(\omega_{R}-\omega_{0}\right) / \delta_{R}\right| \approx 0.5$. Thus, the result that the nature of the $A_{1 g}$ excitation is strictly displacive reflects simply the proximity of $\omega_{0}$ to the resonant maximum. The intermediate behavior found in our experiments is consistent with $\left|\left(\omega_{R}-\omega_{0}\right) / \delta_{R}\right| \approx 2.0$. Since in the two-band case $\partial \chi^{(1)} / \partial \mathcal{Q} \propto \partial \chi^{(1)} / \partial \omega$ where the proportionality constant is either $a$ or $e$ [15], our model carries the predictions that $\Delta R$ should be proportional to the RS cross section (depicted in the inset of Fig. 2) and that, at a given $\omega_{0}$, the relative intensities gained from RS and time-domain data should be the same. The FT spectrum in Fig. 2 gives $|a / e|=3.0 \pm 0.2$ in good agreement with RS measurements. In addition, for a constant pump beam intensity, we calculate that $\Delta R$ at $\omega_{0}=2 \mathrm{eV}$ should be a factor of $\sim 15$ larger than at $\omega_{0}=1.5 \mathrm{eV}$. This value compares favorably with the factor of $\sim 7$ obtained from our experiments and results reported in [4].

The authors are indebted to $\mathrm{C}$. Uher for providing antimony samples and valuable discussions. This work was supported by the NSF through the Center for Ultrafast Optical Science under STC PHY 8920108, and by the U.S. Army Research Office under Contract No. DAAH04-961-0183. T.F. A. was supported in part by the Feodor Lynen Program of the Alexander von Humboldt-Foundation.

Note added.-After this paper was submitted, we became aware of related results by Hase et al. [19] on Bi revealing coherent $E_{g}$ oscillations.

[1] For recent reviews, see S. Ruhman, A. G. Joly, and K. A. Nelson, IEEE J. Quantum Electron. 24, 460 (1988); W. A. Kütt, W. Albrecht, and H. Kurz, IEEE J. Quantum Electron. 28, 2434 (1992).

[2] J. M. Chwalek et al., Appl. Phys. Lett. 57, 1696 (1990).

[3] G. C. Cho, W. Kütt, and H. Kurz, Phys. Rev. Lett. 65, 764 (1990).

[4] T. K. Cheng et al., Appl. Phys. Lett. 59, 1923 (1991); H. J. Zeiger et al., Phys. Rev. B 45, 768 (1992).

[5] T. Pfeifer, W. Kütt, H. Kurz, and R. Scholz, Phys. Rev. Lett. 69, 3248 (1992).

[6] A. Yamamoto et al., Phys. Rev. Lett. 73, 740 (1994).

[7] T. Dekorsky et al., Phys. Rev. Lett. 74, 738 (1995).

[8] Y. Liu et al., Phys. Rev. Lett. 75, 334 (1995).

[9] A. L. Dobryakov et al., JETP Lett. 61, 985 (1995).

[10] D. A. Williams, Phys. Rev. Lett. 69, 2551 (1992).

[11] S. Fahy and R. Merlin, Phys. Rev. Lett. 73, 1122 (1994).

[12] See, e.g., Y. R. Shen and N. Bloembergen, Phys. Rev. 137, A1787 (1965).

[13] R. Scholz, T. Pfeifer, and H. Kurz, Phys. Rev. B 47, 16229 (1993).

[14] A. V. Kuznetsov and C. J. Stanton, Phys. Rev. Lett. 73, 3243 (1994).

[15] J. B. Renucci, W. Richter, M. Cardona, and E. Schönherr, Phys. Status Solidi (b) 60, 299 (1973).

[16] The distinction between $q=0$ and $q \sim \Omega n / c-$ $2 \kappa \omega_{0} / c$ is particularly important for materials without a center of inversion (e.g., GaAs [3] and Te [7]) which show strong dispersion at small wave vectors due to Raman active polaritons.

[17] See, e.g., M. Cardona, in Light Scattering in Solids II, edited by M. Cardona and G. Güntherodt, Topics in Applied Physics Vol. 50 (Springer, Berlin, 1982), Chap. 2.

[18] A related result for molecular systems is given by J. Chesnoy and A. Mokhtari, Phys. Rev. A, 38, 3566 (1988).

[19] M. Hase et al., Appl. Phys. Lett. (to be published). 